\title{
Repetibilidade de caracteres agronômicos em Panicum maximum Jacq. ${ }^{1}$
}

\section{Janaina Azevedo Martuscello ${ }^{2}$, Liana Jank ${ }^{3}$, Dilermando Miranda da Fonseca ${ }^{4}$, Cosme Damião Cruz ${ }^{5}$, Daniel de Noronha Figueiredo Vieira da Cunha ${ }^{2}$}

\author{
${ }^{1}$ Parte da tese da primeira autora. Financiamento: Unipasto e CNPq. \\ 2 Doutorando do Departamento de Zootecnia - Universidade Federal de Viçosa. Bolsista do CNPq. \\ ${ }^{3}$ Embrapa Gado de Corte. Bolsista de Produtividade em Desenvolvimento Tecnológico e Extensão Inovadora do CNPq. \\ ${ }^{4}$ Departamento de Zootecnia - Universidade Federal de Viçosa. \\ ${ }^{5}$ Departamento de Biologia Geral - Universidade Federal de Viçosa.
}

RESUMO - O experimento foi conduzido para avaliar o coeficiente de repetibilidade de algumas características agronômicas em híbridos dePanicum maximum. Dez parcelas de plantas sexuais da espécie foram aleatoriamente distribuídas entre as 230 parcelas de acessos apomíticos. Após a fecundação natural, sementes de cada planta sexual constituíram-se uma família de meios-irmãos. Trinta plantas de cada progenitora foram avaliadas em delineamento de blocos ao acaso, com cinco plantas por parcela e seis repetições. A partir de cinco cortes, estimou-se o coeficiente de repetibilidade pelos métodos da Análise de Variância, Componentes principais (matriz de correlação e covariância) e Análise estrutural. Os coeficientes de repetibilidade (r) nos diferentes métodos, para todas as características avaliadas, oscilaram entre 0,51 e 0,86 e podem ser considerados altos, assim como os coeficientes de determinação. As estimativas do coeficiente de repetibilidade obtidas para as quatro características avaliadas pelo método da análise de variância foram quase sempre menores que as obtidas pelos demais métodos. Pelo método dos componentes principais (baseado na matriz de covariância), as estimativas foram sempre maiores em relação aos demais métodos. Considerando satisfatórios níveis de 80 ou $90 \%$ de confiabilidade para avaliação da superioridade relativa dos híbridos para todas as características avaliadas, as cinco medições realizadas são suficientes para escolha da melhor planta. A exclusão dos cortes 1 e 2 promove aumento nos coeficientes de repetibilidade e determinação.

Palavras-chave: análise estrutural, análise de variância, componentes principais, produção de matéria seca

\section{Agronomic characters repeatability in Panicum maximum Jacq.}

\begin{abstract}
The experiment was carried out to evaluate the coefficient of repeatability of some agronomic characteristics in Panicum maximum hybrids. Ten plots of sexual plants of this species were randomly distributed among 230 plots of apomitic accessions. After natural hybridization, seeds of each sexual plant constituted a half-sib family. Thirty plants of each female progenitor were evaluated in a randomized blocks design, with five plants per plot and six replications. Based on five evaluation cuts, the coefficient of repeatability was estimated by the methods analysis of variance, principal components (correlation and covariance matrices) and structural analyses. The coefficient of repeatability (r) estimated by the different methods, for all the characteristics evaluated, was high and varied from 0.51 to 0.86 . The estimates of the coefficient of determination were also high. It was verified that the estimates of the repeatability coefficient for the four characteristics evaluated by the analyses of variance method were almost always smaller than the estimates obtained by the other methods. By the principal component method (based on the covariance matrix), the estimates were always the highest. Considering a level of 80 or $90 \%$ as satisfactory for the confidence in the decision of the relative superiority of the hybrids based on green matter yield, these five evaluation cuts would be sufficient for the choice of the best plant. The exclusion of the first two cuts will increase the repeatability and determination coefficients.
\end{abstract}

Key Words: analysis of variance, dry matter production, principal components, structural analysis

\section{Introdução}

A área ocupada pela espécie Panicum maximum Jacq. corresponde a aproximadamente $20 \%$ de toda a área de pastagens cultivadas no Brasil (em torno de 100 milhões de hectares) suprindo 30\% do mercado de sementes forrageiras (Abrasem, 2004). Considerando a crescente intensificação dos sistemas de produção de bovinos no Brasil, a espécie P. maximum, pelo seu potencial produtivo e sua qualidade, é uma das mais indicadas para esse tipo de exploração. A disponibilidade de cultivares melhorados, como Tanzânia, Mombaça, Massai e Milênio, tem sido responsável pela ampliação da área de cultivo desta forrageira. A continuação dos trabalhos de melhoramento genético dessa forrageira 
contribuirão significativamente para o aumento da produção de carne, leite, couro e lã no País.

A maioria dos acessos de $P$. maximumtem reprodução do tipo apomítica, ou seja, a planta gerada possui as mesmas características da planta-mãe, portanto, não possui variabilidade genética. Neste caso, então, verifica-se a necessidade de plantas sexuais para intercâmbio gênico (Savidan, 1989). Assim, o cruzamento entre plantas sexuais e apomíticas de $P$. maximum constitui alternativa viável para obtenção de forrageiras superiores quanto à produção vegetal e, conseqüentemente, à produção animal.

Durante o processo de seleção de plantas visando ao lançamento de novos cultivares ou à escolha de genitores superiores para recombinação, é importante certificar-se de sua superioridade genética. A análise de sucessivas medições de uma característica em um grupo de indivíduos é desejável no melhoramento genético de plantas perenes, uma vez que, espera-se, a superioridade ou a inferioridade inicial de um indivíduo mantém-se ao longo das medições. Portanto, coeficiente de repetibilidade valida essa expectativa (Cruz et al., 2004).

Altos valores de repetibilidade para quaisquer características indicam que é possível predizer o valor real dos indivíduos com base em determinado número de medições. Assim, a medida de consistência da posição em relação à classificação dos indivíduos durante sucessivas medições de determinado caráter é denominada repetibilidade (Turner \& Young, 1969). Segundo alguns autores (Lush, 1964; Abeywardena, 1972; Kempthorne, 1973; Cruz et al., 2004), a repetibilidade pode ser definida como a correlação entre sucessivas medidas, obtidas de um mesmo indivíduo, e avaliações tomadas ao longo do tempo ou do espaço.

Quando uma característica possui alta repetibilidade, há indicativo de pouco ganho em acurácia com o aumento do número de medidas repetidas. No entanto, quando a repetibilidade apresenta baixos valores, é necessário grande número de medições para se alcançar valor de determinação satisfatório. Assim, ao aumentar o número de medidas tomadas para determinada característica, diminui-se a variância temporária causada pelo ambiente e, evidentemente, reduz-se também a variância fenotípica, melhorando a acurácia do coeficiente de repetibilidade. Ressalta-se que, quando a variância ambiental temporária é baixa e a repetibilidade é alta, o aumento no número de tomada de dados pouco acrescentará na melhoria da inferência do valor genotípico do indivíduo. No entanto, se o valor da repetibilidade é baixo, aumentando o número de avaliações haverá aumento de ganho.

Neste trabalho objetivou-se determinar os coeficientes de repetibilidade das características produção de matéria verde (MV), produção de matéria seca (MS), produção de matéria seca foliar (MSF) e porcentagem de folhas (\%F) em híbridos de $P$. maximum a partir de dados obtidos durante dois anos (cinco cortes). Estimou-se ainda o número mínimo de medições para a seleção com maior eficiência e confiabilidade e para a estabilização fenotípica da pro gênie.

\section{Material e Métodos}

O experimento foi conduzido na Embrapa Gado de Corte no município de Campo Grande, Mato Grosso do Sul, a 20²7' de latitude e $54^{\circ} 57^{\prime}$ de longitude.O clima, segundo a classificação de Köppen, é do tipo tropical chuvoso de savana, subtipo Aw, caracterizado por distribuição anual irregular das chuvas e pela ocorrência bem definida do período seco durante os meses frios e do período chuvoso durante o verão. Os 250 acessos do banco de germoplasma de Panicum maximum estavam bem conservados em campo e cultivados em parcelas de $4 \times 4 \mathrm{~m}^{2}$ com $2 \mathrm{~m}$ entre elas. Distribuídos aleatoriamente entre os acessos apomíticos (tetraplóides), encontravam-se dez parcelas com plantas sexuais (tetraplóides) que tiveram o número de cromossomos duplicados pela aplicação de colchicina. $\mathrm{Na}$ época de florescimento (março a maio), as plantas sexuais foram fecundadas naturalmente pelos acessos vizinhos, fazendo com que suas sementes gerassem famílias de meios-irmãos. Sementes híbridas de dez plantas sexuais foram colhidas em maio e junho de 2004 e semeadas em outubro em bandejas de isopor com substrato vermiculita:areia 1:1. Após dois meses, 30 plântulas de cada progenitora sexual foram transplantadas para terra em sacos plásticos e mantidas até condições adequadas para plantio no campo.

Em dezembro de 2005, as mudas por progênies foram transplantadas para a área experimental em um delineamento de blocos ao acaso, com cinco plantas por parcela e seis repetições, totalizando 30 plantas por família. O espaçamento foi de $1 \mathrm{~m}$ entre parcelas e entre plantas na parcela e de $2 \mathrm{~m}$ entre repetições. O solo da área experimental, umLatossolo Vermelho Escuro, apresentou as seguintes características: $\mathrm{pH} \mathrm{CaCl}{ }_{2}=4,53 ; \mathrm{P}=1,29 \mathrm{mg} / \mathrm{dm}^{3} ; \mathrm{K}=48,6 \mathrm{mg} / \mathrm{dm}^{3}$; $\mathrm{Ca}^{2+}=1.64 \mathrm{cmol}_{\mathrm{c}} / \mathrm{dm}^{3} ; \mathrm{Mg}^{2+}=0,93 \mathrm{cmol}_{\mathrm{c}} / \mathrm{dm}^{3} ; \mathrm{H}+\mathrm{Al}=$ $\left.8,31 \mathrm{cmol}_{\mathrm{c}} / \mathrm{dm}^{3}\right)$.

No início de novembro de 2005, efetuou-se a aplicação de calcário dolomítico ( $3 \mathrm{t} / \mathrm{ha}$ ) incorporado. As adubações fosfatada e potássica consistiram, respectivamente, de $100 \mathrm{~kg}$ de $\mathrm{P}_{2} \mathrm{O}_{5} / \mathrm{ha}$ (superfosfato simples) e $100 \mathrm{~kg}$ de $\mathrm{K}_{2} \mathrm{O} / \mathrm{ha}$ (cloreto de potássio). Procedeu-se também à aplicação de FTE BR16 (50 kg/ha) e à adubação nitrogenada com $100 \mathrm{~kg}$ de N/ha (uréia) no transplantio. Outra adubação 
idêntica à anterior, à exceção dos 50 kg de FTE BR16/ha, foi realizada em dezembro de 2005.

Foram feitos cinco cortes no período das águas em todas as plantas (3/3/2005, 13/4/2005, 8/12/2005, 24/1/2006 e 8/3/2006). Imediatamente após o corte, o material foi pesado para obtenção da produção de matéria verde (MV). Amostras foram separadas em lâmina, colmo + bainha e material morto para estimativa da produção de MS total e da MSF. Em seguida, determinou-se a \% de folhas de cada genótipo.

O coeficiente de repetibilidade ( $r$ ) foi estimado por quatro procedimentos estatísticos, que possibilitaram avaliar a consistência da estimativa e permitiram conclusões mais confiáveis sobre as características estudadas. Os estimadores dos coeficientes de repetibilidade foram obtidos por meio do método da análise de variância, do método dos componentes principais (baseado na matriz de covariância ou correlação) e da análise estrutural (baseado na matriz de correlação).

Para estimação do coeficiente de repetibilidade pelo método da Análise de Variância (ANOVA), utilizou-se o modelo estatístico com dois fatores de variação:

$$
Y_{i j}=\mu+g_{i}+a_{j}+e_{i j} \text {, }
$$

em que $\mu=$ média geral; $\mathrm{g}_{\mathrm{i}}=$ efeito aleatório do i-ésimo genótipo sob a influência do ambiente permanente; $a_{j}=$ efeito fixo do ambiente temporário na j-ésima medição; $\mathrm{e}_{\mathrm{ij}}=$ efeito do ambiente temporário associado à j-ésima medição no i-ésimo genótipo.

Para esse modelo, o coeficiente de repetibilidade foi determinado por:

$$
\hat{r}=\operatorname{cov}\left(\mathrm{Y}_{i j}, \mathrm{Y}_{i j^{\prime}}\right) / \sqrt{V\left(\mathrm{Y}_{i j}\right) V\left(\mathrm{Y}_{i j^{\prime}}\right)}=\frac{\hat{\sigma_{g}^{2}}}{\hat{\sigma^{2}+\hat{\sigma_{g}^{2}}}}
$$

A metodologia dos componentes principais se baseou na matriz de correlação ou na matriz de covariância entre os genótipos, obtidas em cada par de medições, que estimam de forma mais eficiente o coeficiente de repetibilidade em casos no quais os genótipos apresentam comportamento cíclico em relação ao caráter estudado.

Quando se baseou na matriz de correlações, o coeficiente de repetibilidade foi determinado por:

$$
\hat{r}=\frac{\lambda_{1}-1}{n-1}
$$

em que: $\lambda_{1}=$ autovalor obtido da matriz $\hat{R}$, associado ao autovetor, cujos elementos tiveram o mesmo sinal e magnitudes semelhantes; e $\mathrm{n}=$ número de períodos avaliados.
Quando se baseou na matriz de covariâncias, o coeficiente de repetibilidade foi estimado considerando-se a matriz paramétrica de variâncias e covariância fenotípicas:

$$
\hat{r}=\frac{\lambda-\hat{\sigma}_{y}^{2}}{\hat{\sigma}_{y}^{2}(n-1)}
$$

em que: $\hat{\sigma_{y}^{2}}=\sigma^{2}+\sigma^{2}$

O método da análise estrutural apresenta apenas diferenças conceituais em relação ao método dos componentes principais. Obtiveram-se também as estimativas da repetibilidade por meio da matriz de correlações entre genótipos em cada par de avaliação. Assim,o estimador do coeficiente de repetibilidade foi determinado por:

$$
\hat{r}=\frac{a^{\prime} \hat{R} a-1}{n-1}
$$

em que: a' = autovetor com elementos paramétricos associados ao maior autovalor de $\mathrm{R} ; \mathrm{R}=$ matriz paramétrica de correlação entre genótipos em cada par de avaliação; $\hat{R}=$ estimador da matriz R; e $\mathrm{n}=$ número de períodos avaliados.

Após a obtenção do coeficiente de repetibilidade, estimou-se o número mínimo de avaliações necessárias para predizer o valor real dos indivíduos, com base em um coeficiente de determinação $\left(\mathrm{R}^{2}\right)$ preestabelecido. A predição desse valor foi realizada com base na seguinte expressão:

$$
\mathrm{n}_{0}=\mathrm{R}^{2}(1-\hat{r}) /\left(1-\mathrm{R}^{2}\right) \mathrm{r}
$$

em que: $\mathrm{R}^{2}=\mathrm{nr} / 1+\mathrm{r}(\mathrm{n}-1)$

A estabilização fenotípica das quatro características estudadas nos híbridos de $P$. maximum foi avaliada utilizando-se os métodos dos componentes principais, obtidos a partir da matriz de correlações para as sucessivas medições, considerando-se $2,3 \ldots$ até todas as $n$ avaliações efetuadas. Portanto, foram realizadas n-1 análises e duas medições sucessivas; $n-2$, três avaliações sucessivas até chegar às cinco medições.

Todas as análises estatísticas foram realizadas por meio do programa computacional GENES (Programa Computacional em Genética e Estatística) (Cruz, 2001).

\section{Resultados e Discussão}

Observou-se que os coeficientes de repetibilidade (r) oscilaram entre 0,51 e 0,86 (Tabela 1 ). Os maiores valores foram registrados para a MSF e os menores para produção de MS. No processo de seleção de novas forrageiras, a 
característica produção de MSF, fundamental na alimentação animal, é imprescindível, pois as folhas apresentam maior digestibilidade em relação ao colmo. A seleção com base nessa característica tende a tornar o processo de melhoramento genético mais eficiente, principalmente na etapa final, quando a avaliação do ganho de peso é o principal indicador do potencial de utilização da planta.

As estimativas dos coeficientes de repetibilidade para avaliação das características MV, MS, MSF e \% F, de modo geral, podem ser consideradas altas e indicam a confiabilidade na identificação dos melhores genótipos, considerando as cinco avaliações utilizadas.

Independentemente do método utilizado para estimativa do coeficiente de repetibilidade, os coeficientes de determinação variaram de 83,96 a 96,88\% (Tabela 1) e, portanto, podem ser classificados como altos. Como o coeficiente de determinação expressa a acurácia na predição do valor real do indivíduo, a confiabilidade na seleção das melhores progênies de P. maximum, baseada no valor fenotípico de MSF, é de 96,88\% (Método dos componentes principais baseado na matriz de covariância), portanto, as cinco colheitas foram suficientes para proporcionar alta confiabilidade na predição do comportamento de diferentes genótipos.

As estimativas do coeficiente de repetibilidade obtidas para as quatro características avaliadas pelo método da análise de variância foram quase sempre menores que as obtidas pelos demais métodos avaliados. Pelo método dos componentes principais (baseado na matriz de covariância), essas estimativas foram sempre maiores que as obtidas pelos demais métodos. Ainda assim, para todas as características avaliadas, as estimativas obtidas pelas quatro metodologias utilizadas comprovaram acurácia nas medições realizadas e alta regularidade da superioridade dos indivíduos.
Segundo Abeywardena (1972), o método dos componentes principais permite estimar de forma mais eficiente o coeficiente de repetibilidade em situações em que os genótipos apresentam comportamento cíclico em relação ao caráter estudado, por basear-se na matriz de covariância entre genótipos obtidas em cada par de medições. De acordo com Cruz et al. (2004), o método da análise de variância pode, em alguns casos, levar a estimativas subdimensionadas do coeficiente de repetibilidade.

De modo geral, a diferença entre as estimativas do coeficiente de repetibilidade nos quatro métodos indica a necessidade de utilização de vários métodos disponíveis para se obter um intervalo preciso dentro do qual, com maior probabilidade, seja encontrado o valor real para esse parâmetro.

O aumento da precisão em relação à predição do valor real das avaliações, em todos os métodos utilizados, implicou aumento considerável do número de medições para todas as características (Tabela 2). Esses dados sugerem que a tentativa de aumento de precisão além de $95 \%$ exigiria aumento significativo do número de medições, o que pouco acrescentaria em precisão, tornando seu uso injustificável.

Na seleção de híbridos de $P$. maximum, Resende et al. (2004) obtiveram $80 \%$ de precisão para MSF em quatro anos de avaliação sob cortes, porém com um baixo aumento na eficiência de seleção após três anos de avaliação (15 cortes). Para Brachiaria brizantha, no entanto, Basso (2005) obteve $80 \%$ de precisão para esta variável com 6 a 14 cortes, dependendo dos genótipos avaliados em diferentes campos. Alguns estudos de repetibilidade para outras plantas forrageiras baseados em dados obtidos no Brasil estão disponíveis na literatura (Shimoya et al., 2002; Pereira et al., 2002; Daher et al., 2004; Souza Sobrinho et al., 2004).

O número de cortes necessários para caracterizar as produções de MV e MS com $80 \%$ de probabilidade de seu

Tabela 1 - Estimativas do coeficiente de repetibilidade e do coeficiente de determinação (entre parênteses) das características produção de matéria verde (MV), produção de MS, produção de MS foliar (MSF) e porcentagem total de folhas (\% F) avaliados por quatro métodos

Table 1 - Estimates of the coefficients of repeatability and determination (between brackets) for the characteristics green mass yield (MV), DM yield, leaf DM yield (MSF) and leaf percentage (\% F), evaluated by four methods

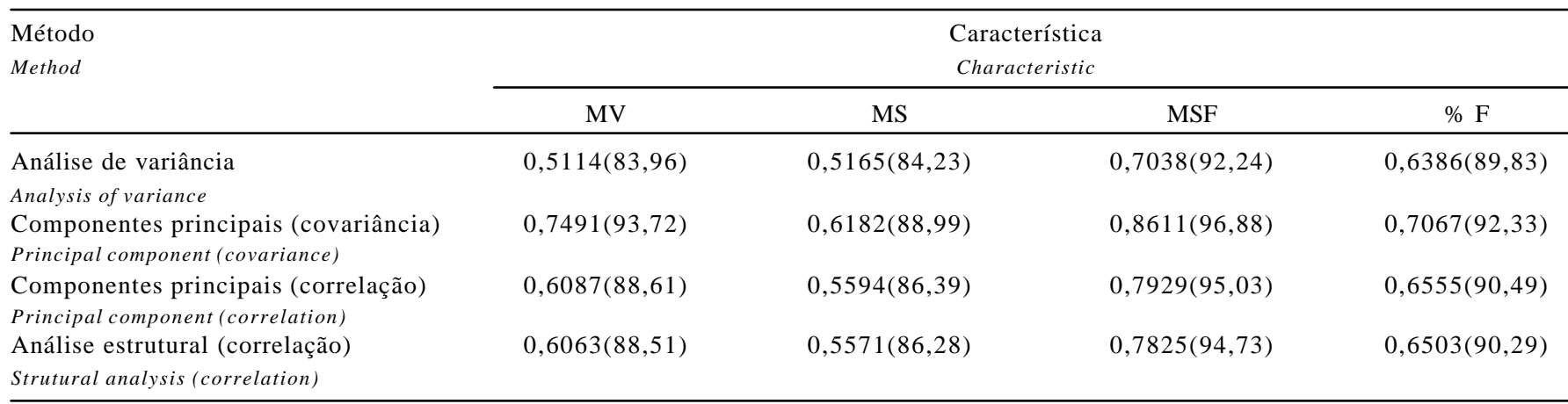


Tabela 2 - Número de medições das características produções de massa verde (MV), matéria seca (MST) e matéria seca foliar (MSF) e porcentagem de folhas (\% folha) em híbridos de Panicum maximum em quatro métodos de estimação do coeficiente de repetibilidade

Table 2 - $\quad$ Number of measurements of the characteristics green mass yield (MV), dry matter yield (MS), leaf dry matter yield (MSF) and leaf percentage (\% F) in Panicum maximum hybrids, associated to different coefficients of determination of the genotypic value $\left(R^{2}\right)$, in four methods of estimation of the coefficient of repeatability

\begin{tabular}{|c|c|c|c|c|}
\hline $\mathrm{R}$ & $\begin{array}{c}\text { Anova } \\
\text { Analysis of variance }\end{array}$ & $\begin{array}{l}\text { Componentes principais (covariância) } \\
\text { Principal components (covariance) }\end{array}$ & $\begin{array}{l}\text { Componentes principais (correlação) } \\
\text { Principal components (correlation) }\end{array}$ & $\begin{array}{c}\text { Análise estrutural (Correlação) } \\
\text { Strutural analysis (correlation) }\end{array}$ \\
\hline
\end{tabular}

Matéria verde (Green matter)

\begin{tabular}{lrrrr}
0,8 & 4 & 1 & 2 & 3 \\
0,85 & 5 & 2 & 3 & 4 \\
0,9 & 9 & 3 & 6 & 12 \\
0,95 & 18 & 6 & 64 & 12 \\
0,99 & 94 & 33 & 64 \\
\hline
\end{tabular}

Matéria seca (Dry matter)

\begin{tabular}{lrrrr}
0,8 & 4 & 2 & 3 & 3 \\
0,85 & 5 & 3 & 4 & 7 \\
0,9 & 8 & 6 & 15 & 7 \\
0,95 & 18 & 12 & 78 & 15 \\
0,99 & 93 & 61 & 79 \\
\hline
\end{tabular}

Matéria seca foliar (Leaf dry matter)

\begin{tabular}{lrrrr}
0,8 & 2 & 1 & 1 & 1 \\
0,85 & 2 & 1 & 1 & 2 \\
0,9 & 4 & 2 & 5 & 3 \\
0,95 & 8 & 3 & 26 & 5 \\
0,99 & 42 & 16 & 27 & 26 \\
\hline
\end{tabular}

$\%$ de folhas (Leaf \%)

\begin{tabular}{lrrrr}
0,8 & 2 & 2 & 2 & 2 \\
0,85 & 3,2 & 2 & 3 & 3 \\
0,9 & 5 & 4 & 5 & 10 \\
0,95 & 11 & 8 & 52 & 10 \\
0,99 & 56 & 41 & 53 \\
\hline
\end{tabular}

valor real foi de no máximo quatro (paro o método da análise de variância) e apenas um e dois, respectivamente, para o método de componentes principais (baseado na matriz de covariância), o que indica não serem necessários cinco cortes para essas características, pois com apenas quatro (para o método que estima o maior número de medições) obtém-se boa confiabilidade, além de redução da mão-deobra e do tempo.

Essa observação é válida também para as demais características avaliadas.Para a produção de MSF e a \% folhas, o número de medições necessárias para os mesmos $80 \%$ de probabilidade é de duas medições, o que indica novamente que os cinco cortes foram suficientes para estimativa confiável do coeficiente de repetibilidade. Para se obter maior confiabilidade, pode-se utilizar o coeficiente de determinação de $90 \%$ para a seleção dos melhores genótipos para todas as características avaliadas. Neste caso, para maior acurácia, são necessários 3 a 9 cortes, de acordo com a metodologia.

A utilização de genótipos estabilizados na obtenção de estimativas de repetibilidade é fundamental. Em alguns casos, é possível um caráter ser regido por conjuntos gênicos distintos e os genes estarem mais ou menos ativos de acordo com o estado de desenvolvimento dos indivíduos. Em alguns casos, a inclusão de avaliações em estádio precoce onde não há plena manifestação do potencial genético da planta, ou em avaliações tardias, quando a forrageira apresenta determinado grau de senescência, a repetibilidade pode ser subestimada. Assim, se o genótipo no qual as medidas são tomadas não estiver estabilizado, a variação dentro de indivíduos incluirá parte considerável da variância da interação genótipo $\times$ ambiente temporário e o aumento do número de medições como forma de reduzir a variação dentro de indivíduos pode não ser vantajoso, pois a variância adicional poderá ser suficiente para neutralizar a redução do componente.

Para a variável produção de MV, o maior coeficiente de repetibilidade foi obtido quando se correlacionaram os cortes 1 e 2 (Tabela 3 ) e o menor, na correlação dos cortes 2 e 3 , o que provavelmente foi ocasionado pelo fato de que, nos dois primeiros cortes, os genótipos ainda não estavam estabilizados. De fato, a produção de matéria 
Tabela 3 - Números de medições ( $n$ ) e obtenção de $R^{2}$ por meio da estabilização fenotípica utilizando-se o método dos componentes principais a partir da matriz de correlação de quatro características agronômicas avaliadas em híbridos dePanicummaximum em cinco sucessivas colheitas

Table 3 - Estimate of the number of measurements ( $n$ ) and obtention of the coefficients of determination of the genotypic value $\left(R^{R}\right)$, by the phenotypic stabilization using the principal components method, based on the correlation matrix of four agronomic characteristics evaluated in hybrids of Panicum maximum in five successive harvests

\begin{tabular}{|c|c|c|c|c|c|c|c|}
\hline \multirow[t]{2}{*}{$\begin{array}{l}\text { Colheita } \\
\text { Harvest }\end{array}$} & $\begin{array}{l}\text { № de medições } \\
\text { Number of harvests }\end{array}$ & $\begin{array}{l}\text { Coeficiente de repetibilidade } \\
\text { Coefficient of repeatability }\end{array}$ & $\mathrm{R}^{2}$ & $\begin{array}{c}\text { Colheita } \\
\text { Harvest }\end{array}$ & $\begin{array}{l}\text { № de medições } \\
\text { Number of harvests }\end{array}$ & $\begin{array}{l}\text { Coeficiente de repetibilidade } \\
\text { Coefficient of repeatability }\end{array}$ & $\mathrm{R}^{2}$ \\
\hline & \multicolumn{3}{|c|}{ Matéria verde } & \multicolumn{4}{|c|}{$\begin{array}{l}\text { Matéria seca foliar } \\
\text { Leaf dry matter yield }\end{array}$} \\
\hline $1-2$ & 2 & 0,92 & 95 & $1-2$ & 2 & 0,52 & 69 \\
\hline $2-3$ & 2 & 0,35 & 52 & $2-3$ & 2 & 0,61 & 76 \\
\hline $3-4$ & 2 & 0,90 & 95 & $3-4$ & 2 & 0,89 & 94 \\
\hline $4-5$ & 2 & 0,69 & 82 & $4-5$ & 2 & 0,92 & 96 \\
\hline 1,2 e 3 & 3 & 0,62 & 83 & 1,2 e 3 & 3 & 0,68 & 87 \\
\hline 2,3 e 4 & 3 & 0,57 & 80 & 2,3 e 4 & 3 & 0,71 & 88 \\
\hline 3,4 , e 5 & 3 & 0,75 & 90 & 3,4 , e 5 & 3 & 0,92 & 97 \\
\hline $1,2,3$ e 4 & 4 & 0,60 & 86 & $1,2,3$ e 4 & 4 & 0,75 & 92 \\
\hline $2,3,4$ e 5 & 4 & 0,59 & 85 & $2,3,4$ e 5 & 4 & 0,77 & 93 \\
\hline \multirow[t]{3}{*}{$1,2,3,4$ e 5} & 5 & 0,61 & 89 & $1,2,3,4$ e 5 & 5 & 0,79 & 95 \\
\hline & \multirow{2}{*}{\multicolumn{2}{|c|}{$\begin{array}{l}\text { Matéria seca } \\
\text { Dry matter }\end{array}$}} & & \multirow{2}{*}{\multicolumn{4}{|c|}{$\begin{array}{l}\% \text { folhas } \\
\text { Leaf } \%\end{array}$}} \\
\hline & & & & & & & \\
\hline $1-2$ & 2 & 0,85 & 92 & $1-2$ & 2 & 0,86 & 93 \\
\hline $2-3$ & 2 & 0,36 & 54 & $2-3$ & 2 & 0,51 & 67 \\
\hline $3-4$ & 2 & 0,86 & 92 & $3-4$ & 2 & 0,97 & 98 \\
\hline $4-5$ & 2 & 0,58 & 73 & $4-5$ & 2 & 0,71 & 83 \\
\hline 1,2 e 3 & 3 & 0,58 & 81 & 1,2 e 3 & 3 & 0,68 & 87 \\
\hline 2,3 e 4 & 3 & 0,57 & 80 & 2,3 e 4 & 3 & 0,66 & 86 \\
\hline 3,4 , e 5 & 3 & 0,64 & 84 & 3,4 , e 5 & 3 & 0,77 & 91 \\
\hline $1,2,3$ e 4 & 4 & 0,58 & 85 & $1,2,3$ e 4 & 4 & 0,70 & 90 \\
\hline $2,3,4$ e 5 & 4 & 0,53 & 82 & $2,3,4$ e 5 & 4 & 0,62 & 87 \\
\hline $1,2,3,4$ e 5 & 5 & 0,56 & 87 & $1,2,3,4$ e 5 & 5 & 0,65 & 90 \\
\hline
\end{tabular}

verde foi a de maior instabilidade. Esse resultado era previsível, uma vez que a teor de água em cada planta tem reflexo direto nessa estimativa. Não só para MV, mas também para MS, a baixa correlação entre os cortes 2 e 3 pode ser explicada pela época do corte, pois o terceiro corte foi realizado em dezembro e as plantas se desenvolveram após rebrotação de corte na seca. Para MS o maior coeficiente de repetibilidade e o maior coeficiente de determinação estiveram correlacionados entre os cortes 3 e 4 , provavelmente em razão das condições ambientais, que foram bastante homogêneas quanto à temperatura e à precipitação.

A correlação entre os cortes e a estabilização do genótipo para a produção de MSF e a porcentagem de folhas provavelmente sofreu efeito direto da época de florescimento de cada progênie, uma vez que em florescimento ocorre maior acúmulo de colmo e, conseqüentemente, efeito direto desse acúmulo sobre a produção de MSF e sobre a porcentagem de folhas em cada genótipo. Para MSF, o maior coeficiente de repetibilidade foi na correlação entre os cortes 4 e 5 e o menor nas correlações entre os cortes 2 e 3 (Tabela 3 ). A correlação entre os cortes 3 e 4 para a porcentagem de folhas, assim como para produção de MS, apresentou maiores coeficientes de repetibilidade e determinação.

Apesar da regularidade no comportamento dos indivíduos nos cortes 1 e 2 (Tabela 3), em geral, a inclusão dos dados a partir desses cortes tende a diminuir o coeficiente de repetibilidade, evidenciando que a estabilização de genótipos de $P$. maximum ocorre provavelmente após o segundo corte, o que sugere a exclusão dos dados dos dois primeiros cortes em futuras avaliações.

\section{Conclusões}

Os coeficientes de repetibilidade, assim como os coeficientes de determinação obtidos para as características agronômicas produção de matéria verde, produção de MS, produção de MSF e porcentagem de folhas em genótipos de $P$. maximum, podem ser considerados altos para todas as características em todos os métodos avaliados.

Para maior acurácia ( $90 \%$ de confiabilidade) na seleção dos melhores híbridos, são necessárias três (método dos componentes principais, baseado na matriz de correlação) a nove (método da análise de variância) medições. 
A exclusão dos cortes 1 e 2 promove aumento nos coeficientes de repetibilidade e determinação. Énecessário utilizar os vários métodos de análise disponíveis para se obter intervalo preciso para cada parâmetro visando encontrar o valor real com maior probabilidade.

\section{Literatura Citada}

ABEYWARDENA, V. An application of principal component analysis in genetics. Journal Genetics, v.16, p.27, 1972.

ASSOCIAÇÃO BRASILEIRA DE SEMENTES - ABRASEM. Disponível em: http://<www.abrasem.br.> Acesso em 1/12/2004.

BASSO, K.C. Estimação de parâmetros genéticos e índice de seleção em genótipos de Brachiaria brizantha. Dourados: Universidade Federal de Mato Grosso do Sul, 2005. 63p Dissertação (Mestrado em Produção Vegetal) - Universidade Federal de Mato Grosso do Sul, 2005.

CRUZ, C.D. Programa Genes Versão Windons: aplicativo computacional em genética e estatística. Viçosa, MG: Universidade Federal de Viçosa, 2001. 684p.

CRUZ, C.D.; REGAZZI, A.J.; CARNEIRO, P.C.S. Modelos biométricos aplicados ao melhoramento genético. 3.ed. Viçosa, MG: Universidade Federal de Viçosa, 2004. v.1. 480p.

DAHER, R.F.; VASQUEZ, H.M.; PEREIRA, A.V. et al. Estimativas de parâmetros genéticos e de coeficientes de repetibilidade de caracteres forrageiros em clones de capimelefante (Pennisetum purpureum Schum). Acta Scientiarum, v.26, n.4, p.483-490, 2004
KEMPTHORNE, O. The design and analysis of experiments. New York: Robert E. Krieger Publishing Company, 1973. 631p.

LUSH, J.L. Melhoramento dos animais domésticos. Tradução de CARNEIRO, G.G.; MEMORIA, J.M.P.; DRUNMOND, G. (Eds.) Rio de Janeiro: CEDEGRA, 1964. 566p.

PEREIRA, A.V.; CRUZ, C.D; FERREIRA, R.P. et al. Influência da estabilização de genótipos sobre a estimativa de repetibilidade em caracteres produtivos de capim-elefante. Ciência e Agrotecnologia, v.26, n.4, p.762-767, 2002.

RESENDE, R.M.S.; JANK, L.; VALLE, C.B. et al. Biometrical analysis and selection of tetraploid progenies of "Panicum maximum" Jacq. using mixed model methodology. Pesquisa Agropecuária Brasileira, v.39, n.4, p.335-341, 2004.

SAVIDAN, Y.H.; JANK, L.; COSTA, J.C.G. et al. Breeding Panicum maximum in Brazil: 1. Genetic resourses, modes of reproduction and breeding procedures. Euphytica, v.41, p.107-112, 1989.

SHIMOYA, A.; PEREIRA, A.V.; FERREIRA, R.P. et al. Repetibilidade de características forrageiras de capim-elefante. Scientia Agricola, v.59, n.2, p.227-234, 2002.

SOUZA SOBRINHO, F.; LEDO, F.J.S.; PEREIRA, A.V. et al. Estimativas de repetibilidade para produção de matéria seca em alfafa. Ciência Rural, v.34, n.2, p.531-537, 2004.

TURNER, H.N.; YOUNG, S.Y. Quantitative gentics insheep breeding. Ithaca: Cormell University Press, 1969. 331p. 